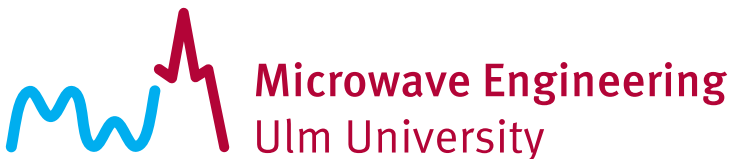

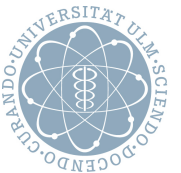

\section{Phase Noise Mitigation for Multistatic FMCW Radar Sensor Networks Using Carrier Transmission}

André Dürr, Benedikt Schweizer, Jonathan Bechter, and Christian Waldschmidt

(C) 2018 IEEE. Personal use of this material is permitted. Permission from IEEE must be obtained for all other uses, in any current or future media, including reprinting/republishing this material for advertising or promotional purposes, creating new collective works, for resale or redistribution to servers or lists, or reuse of any copyrighted component of this work in other works.

DOI: 10.1109/LMWC.2018.2878983 


\title{
Phase Noise Mitigation for Multistatic FMCW Radar Sensor Networks using Carrier Transmission
}

\author{
André Dürr, Benedikt Schweizer, Jonathan Bechter, and Christian Waldschmidt
}

\begin{abstract}
For incoherent radar sensor networks it is desirable to perform both monostatic and bistatic distance measurements. Especially the bistatic measurements enable to achieve additional information about a target. As for bistatic measurements different local oscillators (LO) are used for system operation, the phase noise $(P N)$ at the receiving $(\mathrm{RX})$ sensor node is uncorrelated to the PN of the transmitter (TX). This effect usually degrades the signal-to-noise ratio (SNR) for bistatic measurements in comparison to monostatic measurements. This letter briefly discusses the problem of PN correlation in bistatic distance measurements and proposes a novel system approach that mitigates phase errors between TX and RX oscillators. The proposed concept is mathematically derived and proven with measurements using a radar demonstrator at $77 \mathrm{GHz}$.
\end{abstract}

Index Terms-Millimeter wave radar, FMCW radar, phase noise, multistatic radar, novel radar system, PLL.

\section{INTRODUCTION}

Above the $100 \mathrm{GHz}$ range it is desirable to integrate voltagecontrolled oscillators (VCO), amplifiers, mixers, couplers, and antennas on a single monolithic microwave integrated circuit (MMIC). This approach avoids lossy wave propagation on printed circuit boards (PCB) and shrinks sensor size, which enables new applications [1].

A promising new approach are multisensor networks consisting of many inexpensive radar sensor nodes. This allows to gain more information of the scene under investigation by not only evaluating the monostatic radar response of each target, but also the path between different sensors, i.e., the bistatic response. Usually the TX signals are generated separately on each MMIC. For monostatic measurements a range correlation effect occurs as the TX and the RX signal are correlated to each other [2]. In contrast, bistatic measurements are dramatically affected by PN as the LO signals are generated individually on each MMIC. Thus, very challenging requirements in PN performance of the TX signals have to be fulfilled to be able to evaluate bistatic distance measurements [3], [4]. Recent works also showed that it is possible to achieve signal coherency using adapted modulations and signal processing algorithms. This also enables to realize Multiple Input Multiple Output (MIMO) radar systems but at the expense of SNR reduction in comparison to PN coherent systems [4]-[6].

Inspired by very classical broadcasting techniques using amplitude modulation (AM) with carrier transmission, a new system architecture with carrier transmission is proposed for FMCW radar to overcome these problems. The concept is mathematically derived and proven by radar measurements.

Manuscript received October 2, 2018; accepted October 27, 2018. This work was funded by the Deutsche Forschungsgemeinschaft (DFG, Germany Research Foundation) - WA 3506/8-1. The authors are with the Institute of Microwave Engineering, Ulm University, 89081 Ulm, Germany (e-mail: andre.duerr@uni-ulm.de).

\section{SYSTEM CONCEPT}

For conventional monostatic FMCW radar sensors the same LO is used for the TX and the RX signal. As the RX signal is affected by a time delay within the radar channel, the RX signal is partly correlated to the TX signal. Therefore, a range correlation effect depending on the time delay occurs. The resulting PN power density at the receiver output spectrum can be calculated according to [2] as

$$
\mathcal{L}_{\Delta \Phi}(f)=2 \mathcal{L}_{\Phi}(f)(1-\cos (2 \pi f \tau)) .
$$

$\mathcal{L}_{\Phi}$ describes the PN power density of the TX signal, $f$ the frequency offset from the carrier, $\tau$ the time delay in the radar channel, and $\mathcal{L}_{\Delta \Phi}$ the resulting intermediate frequency (IF) PN power density. The range correlation effect causes that the residual PN around the target is strongly reduced. For bistatic FMCW radar sensor networks in comparison no range correlation effect occurs as different LOs with uncorrelated PN statistics are involved for system operation. For that cases a PN skirt appears around the target leading to a loss in SNR.

The basic principle of the novel system approach is depicted in Fig. 1. In comparison to common FMCW radars, this

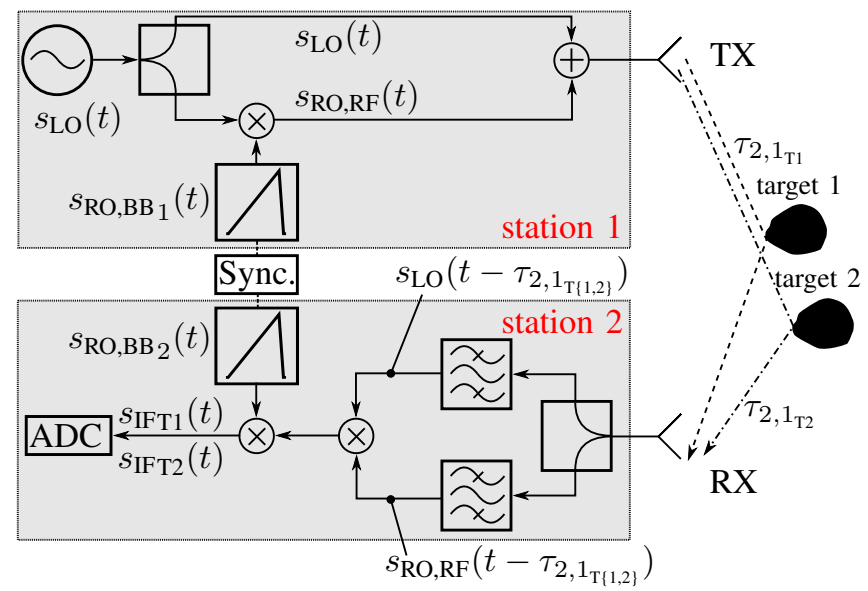

Fig. 1. Novel proposed system setup for a bistatic measurement between station 1 and station 2 . The FMCW signal generation is loosely synchronized with a trigger signal.

system approach also transmits the LO. It is assumed that the FMCW signal is generated at a low frequency in baseband with superior PN characteristics in comparison to the fixed frequency LO, which is used for up-conversion into the radio frequency (RF) band. At the up-converting mixer the PN of both sources add up. Therefore, the inferior PN statistics of the LO is dominating and is therefore mainly present at the RF FMCW signal. This means that the LO and the RF FMCW signal contain the same PN statistics. The LO and the RF FMCW signal do not overlap in frequency domain. Both 
signals are combined and afterwards transmitted. Within the radar channel they are affected by the same channel delay. At the receiver both signals are separated from each other by adequate filtering. The starting frequency of the baseband FMCW signal determines the gap between both signals and has to be chosen such that a practical filter design can be realized. Afterwards, the received RF FMCW signal is downconverted to the baseband using the received LO carrier. In this mixing step the PN cancels out. The IF frequency is obtained by mixing the down-converted RF FMCW signal by the same baseband FMCW signal, which was also used for generating the TX signal. This approach does not require a coherent coupling on a hardware level, only the low frequency FMCW signal generation needs to be synchronized. This can be achieved by using appropriate TX starting frequencies or a time delay $\Delta t$, which can be estimated by an adequate signal processing [4], [5].

\section{PN CANCELlation Signal Model}

The functional principle of the novel PN canceling system concept is mathematically derived for two targets of same intensity and for a bistatic measurement from station 1 to 2 .

The baseband FMCW signal $s_{\mathrm{RO}, \mathrm{BB}_{1}}$ at station 1 is starting with an offset frequency $f_{\text {off }}$

$$
s_{\mathrm{RO}_{, \mathrm{BB}}}(t)=\cos \left(2 \pi\left(f_{\text {off }} t+\frac{B}{2 T} t^{2}\right)+\varphi_{\mathrm{s}, \mathrm{RO}_{1}}\right) .
$$

In (2) $t \in[0 ; T]$ denotes the continuous time with the sweep duration $T, B$ the sweep bandwidth of the FMCW signal, and $\varphi_{\mathrm{s}, \mathrm{RO}_{1}}$ the starting phase. The frequency offset $f_{\text {off }}$ determines the frequency gap between both signals and is necessary to separate the FMCW signal from the LO in the RF band. For simplicity the amplitudes of the TX signals are set to 1 .

The baseband FMCW signal is assumed to be almost perfect in comparison to the LO carrier, i.e., a PN term is neglected here. The LO at high frequencies has inferior $\mathrm{PN}$ performance in comparison to the baseband FMCW signal and can be expressed by

$$
s_{\mathrm{LO}}(t)=\cos \left(2 \pi f_{0} t+\varphi_{\mathrm{s}, \mathrm{LO}}+\phi_{\mathrm{LO}}(t)\right) .
$$

In (3) $f_{0}$ is the LO frequency, $\varphi_{\mathrm{s}, \mathrm{LO}}$ the starting phase, and $\phi_{\mathrm{LO}}$ the PN term. After the mixer only the PN of the LO is present. Therefore, the RF FMCW signal is

$$
\begin{aligned}
& s_{\mathrm{RO}, \mathrm{RF}}(t)=s_{\mathrm{LO}}(t) s_{\mathrm{RO}_{\mathrm{BB}}}(t) \\
& =\cos \left(2 \pi\left(\left(f_{\mathrm{off}}+f_{0}\right) t+\frac{B}{2 T} t^{2}\right)+\varphi_{\mathrm{s}}+\phi_{\mathrm{LO}}(t)\right),
\end{aligned}
$$

with $\varphi_{\mathrm{s}}=\varphi_{\mathrm{s}, \mathrm{RO}_{1}}+\varphi_{\mathrm{s}, \mathrm{LO}}$. The RF FMCW signal is orthogonal in frequency domain with respect to the LO carrier due to the usage of the offset frequency in baseband. They are now combined and then transmitted. The transmitted signal travels to both targets and is partly reflected. At the receiver the LO and the FMCW TX signal are received with a time delay $\tau_{2,1_{\mathrm{T} 1}}$ and $\tau_{2,1_{\mathrm{T} 2}}$, respectively. Both targets are assumed to have the same amplitude, i.e, $A_{1}=A_{2}=A$. The received signals for target 1 and target 2 are

$$
\begin{aligned}
& s_{\mathrm{RX}}(t)=A\left(s_{\mathrm{RO}, \mathrm{RF}}\left(t-\tau_{2,1_{\mathrm{T} 1}}\right)+s_{\mathrm{LO}}\left(t-\tau_{2,1_{\mathrm{T} 1}}\right)\right), \\
& s_{\mathrm{RX}_{\mathrm{T} 2}}(t)=A\left(s_{\mathrm{RO}, \mathrm{RF}}\left(t-\tau_{2,1_{\mathrm{T} 2}}\right)+s_{\mathrm{LO}}\left(t-\tau_{2,1_{\mathrm{T} 2}}\right)\right) .
\end{aligned}
$$

Then the RF FCMW signal is separated from the LO by appropriate filtering. The filtered received FMCW signal at station 2 for target 1 is given by

$$
\begin{aligned}
& s_{\mathrm{RO}, \mathrm{RX}}(t)=A s_{\mathrm{RO}, \mathrm{RF}}\left(t-\tau_{2,1_{\mathrm{T} 1}}\right) \\
& =A \cos \left(2 \pi\left(\left(f_{\mathrm{off}}+f_{0}\right)\left(t-\tau_{2,1_{\mathrm{T} 1}}\right)+\frac{B}{2 T}\left(t-\tau_{2,1_{\mathrm{T} 1}}\right)^{2}\right)\right. \\
& \left.\quad+\varphi_{\mathrm{s}}+\phi_{\mathrm{LO}}\left(t-\tau_{2,1_{\mathrm{T} 1}}\right)\right)
\end{aligned}
$$

The corresponding received and delayed LOs are superimposing at the RX. Using $\cos (x)+\cos (y)=$ $2 \cos \left(\frac{x}{2}-\frac{y}{2}\right) \cos \left(\frac{x}{2}+\frac{y}{2}\right)$ and neglecting the amplitude term, the total received $\mathrm{LO}$ signal at station 2 is given by

$$
\begin{aligned}
s_{\mathrm{LO}, \mathrm{tot}}(t) & =s_{\mathrm{LO}}\left(t-\tau_{2,1_{\mathrm{T} 1}}\right)+s_{\mathrm{LO}}\left(t-\tau_{2,1_{\mathrm{T} 2}}\right) \\
& \propto \cos \left(2 \pi f_{0} t-\pi f_{0}\left(\tau_{2,1_{\mathrm{T} 1}}+\tau_{2,1_{\mathrm{T} 2}}\right)\right. \\
& \left.+\varphi_{\mathrm{s}, \mathrm{LO}}+\frac{\phi_{\mathrm{LO}}\left(t-\tau_{2,1_{\mathrm{T} 1}}\right)}{2}+\frac{\phi_{\mathrm{LO}}\left(t-\tau_{2,1_{\mathrm{T} 2}}\right)}{2}\right),
\end{aligned}
$$

which contains the oscillation and the PN statistics of the TX signal. For two closely spaced targets the PN term is highly correlated, i.e., $\phi_{\mathrm{LO}}\left(t-\tau_{2,1_{\mathrm{T} 2}}\right) \approx \phi_{\mathrm{LO}}\left(t-\tau_{2,1_{\mathrm{T} 1}}\right)$. If the signal in (6) is now down-converted with the signal in (7) the PN of the TX signal cancels out. If they are further apart the range correlation effect according to (1) occurs, but the round-trip delay $\tau$ must be replaced by the inter-target delay $\tau_{2,1_{\mathrm{T} 2}}-\tau_{2,1_{\mathrm{T} 1}}$. Hence, the effectiveness of the new concept depends on the intensities of the targets and the inter-target distance. The concept can be extended to more than two targets. The most challenging case occurs when there are two (see above) or more targets of the same amplitude, as they might interfere destructively. For more than two targets no analytic expression is available but the derivation in (7) can be modeled graphically by an addition of a set of LO vectors in the complex plane. The subsequent signal processing steps are well known and the same as for standard FMCW radar [7].

\section{Measurement Setup}

To verify the novel system approach and to verify the theory, measurements were taken in an anechoic chamber. In order to model a bistatic radar system, a digital radar demonstrator at $77 \mathrm{GHz}$ was used. The block chart is shown in Fig. 2.

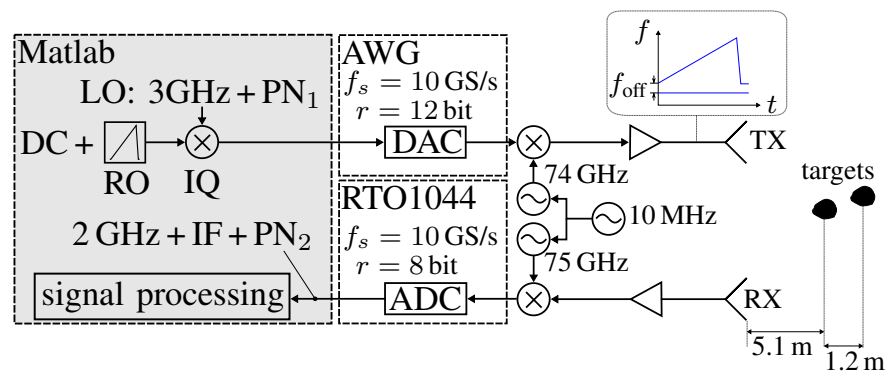

Fig. 2. Block chart of the used measurement setup.

To evaluate the concept it is compared to convential FMCW measurements without carrier transmission. The TX signals 
with the same signal power but with increasing PN power were generated using MATLAB and an arbitrary waveform generator (AWG). In a first step an ideal chirp sequence signal with dc content was generated. Additionally, an ideal LO signal with $f_{0}=3 \mathrm{GHz}$ was generated. This signal was modified with different additional spectral phase locked loop (PLL) PN power densities $\mathcal{L}(f)$ according to [8], which were defined in frequency domain according to a typical PLLs PN spectrum profile $\mathcal{L}(f)$. The $\mathrm{PN}$ spectrum $\mathcal{L}(f)$ is characterized by the $1 / f$ corner frequency $f_{\mathrm{c}}$, the loop filter bandwidth $B_{\mathrm{PLL}}$, the in-band PN level $\mathcal{L}_{0}$, and the thermal noise floor level $\mathcal{L}_{\text {floor }}$. The time domain-jitter can be obtained by an IDFT of $\mathcal{L}(f)$ with uniformly distributed randomized phases [8]. This is then added numerically to the time domain samples of the baseband LO signal. The in-band noise level $\mathcal{L}(f)$ was changed whereas the other parameters remained fixed. A summary of the used signal and modulation parameters can be found in Table I.

TABLE I

Signal And Modulation Parameters for Measurement

\begin{tabular}{l|l} 
Ramp duration $T$ & $100 \mu \mathrm{s}$ \\
Sweep bandwidth $B$ & $800 \mathrm{MHz}$ \\
Number $N$ of frequency chirps & 64 \\
Offset frequency $f_{\text {off }}$ & $200 \mathrm{MHz}$ \\
In-band noise level $\mathcal{L}_{0}$ & $-40 \ldots-60 \mathrm{dBc} / \mathrm{Hz}$ \\
$1 / f$-corner frequency $f_{\mathrm{c}}$ & $1 \mathrm{kHz}$ \\
PLL loopfilter bandwidth $B_{\text {PLL }}$ & $1 \mathrm{MHz}$
\end{tabular}

For more details to the PN model the reader is referred to [8]. After sampling the received radar signal all system operations according to Fig. 1, consisting of bandpass and lowpass filtering, mixing, and FFT, were conducted in MATLAB.

\section{Measurement Results}

The measurements compare the new concept to the standard chirp sequence (CS) approach using a two target scenario. Fig. 3 (a) shows the baseband IF frequency signal for the conventional CS measurements, whereas Fig. 3 (b) shows the baseband IF frequency signal for the new system approach in comparison. For the conventional CS measurements with coherent signal generation but with different LO realizations the noise floor raises with increased PN level $\mathcal{L}_{0}$. Additionally, the target peak powers decrease and the noise floor increases. For very bad PN level $\mathcal{L}_{0}$ the targets are vanishing. The new approach stays nearly unaffected by decreasing PN performance of the TX signal as the range correlation effect of (1) is improved. All IF frequency signals possess similar performance including the same target and noise power levels. Even the target which disappeared for normal CS evaluation becomes visible again with almost the same signal quality.

\section{CONCLUSiON}

A novel FMCW radar approach using frequency orthogonal carrier transmission to mitigate $\mathrm{PN}$ in radar sensor networks was shown. The FMCW signal was generated in baseband with superior PN in comparison to the high frequency LO. A mathematical derivation was shown for a two target scenario. Radar measurements showed that the novel system approach is

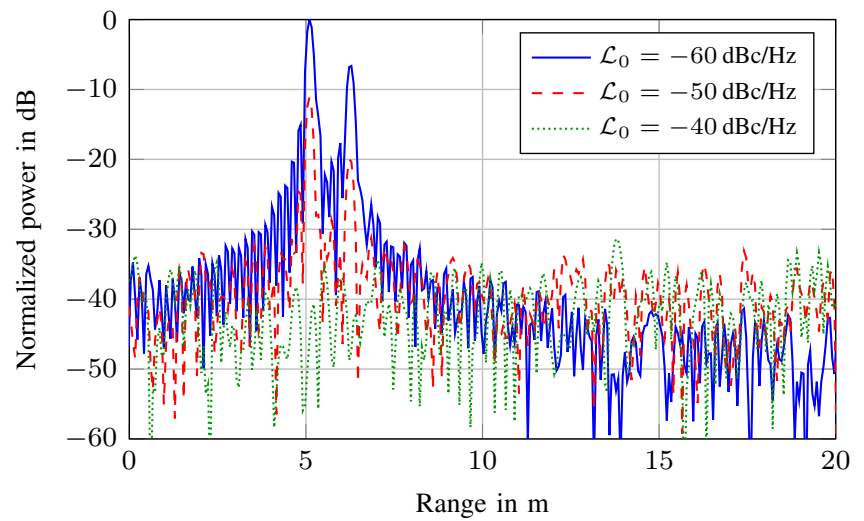

(a) Chirp Sequence evaluation

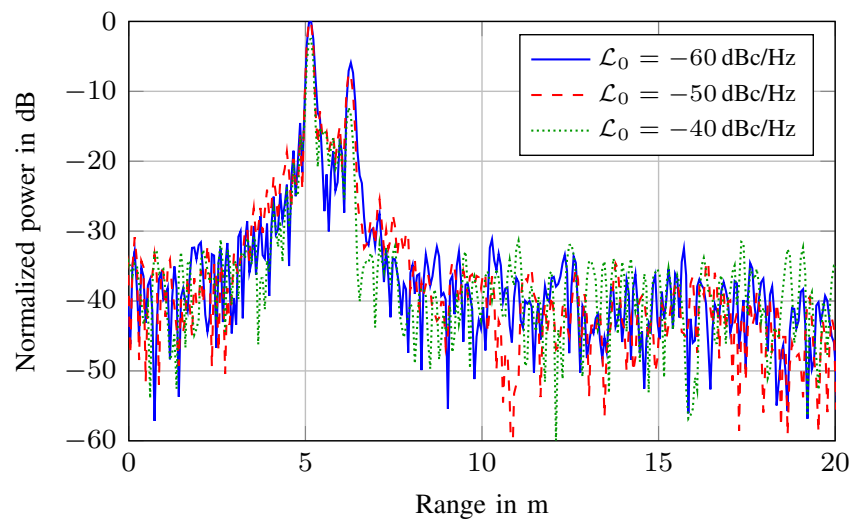

(b) Novel system approach evaluation

Fig. 3. Normalized power of the measured and averaged (64 ramps) IF frequency signal of the standard chirp sequence approach (a) and the novel approach (b) for two static targets placed at $R_{1}=5.1 \mathrm{~m}$ and $R_{2}=6.3 \mathrm{~m}$. The FFT was used with zeropadding $(\times 3)$ and a rectangular window. The in-band PN level $\mathcal{L}_{0}$ from $1 \mathrm{kHz}$ to $1 \mathrm{MHz}$ was adapted between the measurements.

unaffected from PN and has superior performance in comparison to the common FMCW radar approach. The novel concept can establish PN coherency at the RX station and is immune to long-term frequency drift of the LOs. This makes it a suitable candidate for high frequency multistatic sensor networks with challenging constraints in PN performance.

\section{REFERENCES}

[1] R. Lachner, "Industrialization of mmWave SiGe Technologies: Status, Future Requirements and Challenges," in IEEE 13th Topical Meeting on Silicon Monolithic Integrated Circuits in RF Systems, Jan. 2013.

[2] M. C. Budge and M. P. Burt, "Range correlation effects in radars," in Record of the IEEE National Radar Conference, 1993.

[3] A. Frischen et al., "Performance Degradation in Cooperative Radar Sensor Systems due to Uncorrelated Phase Noise," in 11th European Radar Conference, Oct. 2014, pp. 241-244.

[4] A. Frischen, J. Hasch and C. Waldschmidt, "A Cooperative MIMO Radar Network Using Highly Integrated FMCW Radar Sensors," IEEE Transactions on Microwave Theory and Techniques, vol. 65, no. 4, pp. 1355-1366, Apr. 2017.

[5] R. Feger et al., "A 77-GHz FMCW MIMO Radar Based on Loosely Coupled Stations," in German Microwave Conference, Mar. 2012.

[6] A. M. Haimovich et al., "MIMO Radar with Widely Separated Antennas," IEEE Signal Processing Magazine, vol. 25, no. 1, pp. 116-129, 2008.

[7] V. Winkler, "Range Doppler Detection for automotive FMCW Radars," in European Radar Conference, Oct. 2007, pp. 166-169.

[8] L. Smaini, RF Analog Impairments Modeling for Communication Systems Simulation - Application to OFDM based transceivers. Wiley, 2012. 